\title{
Packing effects on polymerization of diacetylene lipids in liposomes and monolayers matrices
}

\author{
Siyeon Baek ${ }^{1}$, Minh Dinh Phan ${ }^{1}$, Jumi Lee ${ }^{1}$ and Kwanwoo Shin \\ Polymerization of 1,2-bis(tricosa-10,12-dinoyl)-sn-glycero-3-phosphocholine, $\mathrm{DC}_{8.9} \mathrm{PC}$, which is a photo-polymerizable lipid, has \\ been systematically investigated in various biological membrane-mimicking models as hosting matrices, for example, small \\ unilamellar vesicles, giant unilamellar vesicles and Langmuir monolayers. In liposome systems, it has been observed that the \\ photo-crosslinking $(\mathrm{PCL})$ reaction between $\mathrm{DC}_{8.9} \mathrm{PC}$ s occurred only in gel phases composed of saturated lipids. No reaction was \\ observed when $\mathrm{DC}_{8.9} \mathrm{PC}$ s were mixed with unsaturated lipids that intrinsically form a liquid phase, even when a high \\ concentration of $\mathrm{DC}_{8.9} \mathrm{PC}$ was added. To explain how the existence of the gel phase-matrix induces polymerization, a mixed \\ Langmuir monolayer system between $\mathrm{DC}_{8.9} \mathrm{PC}$ and 1,2-dipalmitoyl-sn-glycero-3-phosphocholine (DPPC), where the lipid phases \\ can be controlled, has been used. We found that the PCL reaction only occurred at liquid-condensed (LC) phases, and the \\ reaction rates were strongly dependent on the packing states of the monolayers. Therefore, $\mathrm{DC}_{8.9} \mathrm{PCs}$ were possibly distributed in \\ the LC domains of DPPCs, which consequently made the cross-distance between $\mathrm{DC}_{8.9} \mathrm{PC}$ s sufficiently small for the PCL \\ reaction to proceed. \\ Polymer Journal (2016) 48, 457-463; doi:10.1038/pj.2015.136; published online 20 January 2016
}

\section{INTRODUCTION}

Polymerization of photo-polymerizable lipids in assembled systems has been of ongoing interest since the early 1980s because of their potential applications in many fields, such as their use as fundamental platforms for the molecular structure and dynamics of biological membranes, ${ }^{1,2}$ in stabilizing vesicle membranes for biological mimicking models ${ }^{3}$ and for drug carriers and controlled release systems ${ }^{4}$ or in layers to protect electronic devices ${ }^{5}$ from the nonspecific adsorption of proteins, which may cause 'fouling' on the surface of the device. ${ }^{6}$ The thermodynamics and kinetics of the photo-crosslink (PCL) reaction of polymerizable lipids are complicated and depend on many structural, physiological and physical properties of the hosting matrices and their constituent materials. Therefore, it is essential to qualitatively and quantitatively understand the effect of each factor that may impact the application for the controlled system of interest, for example, the controlled release and drug delivery systems.

Depending on the desired features of final products, for example, chemical, mechanical and/or thermal stability, a variety of functionalized polymerizable segments have been incorporated into the lipid structures, mostly into the hydrocarbon chains. The diacetylenefunctionalized chains based on styrene, butadiene and thiol, and the alkene-functionalized chains based on acryloyl, methacryloyl, sorbyl and dienoyl are two common classes for these segments. The basic difference between the chains lies in media-dependent polymerization, ${ }^{7}$ where diacetylene-containing lipids can only be polymerized in the gel phase and consequently exhibit slow kinetics, ${ }^{2}$ while the alkene-containing lipids can be polymerized in either the gel or liquid crystalline phase and yield a higher degree of conversion. ${ }^{8}$ For fundamental studies, our research efforts have focused on diacetylene lipids, in particular 1,2-bis(tricosa-10,12dinoyl)-sn-glycero-3-phosphocholine $\left(\mathrm{DC}_{8,9} \mathrm{PC}\right)$. Studies of small vesicle systems reported that either the crystallization and formation of tubules ${ }^{9}$ or polymerization of $\mathrm{DC}_{8,9} \mathrm{PC}$ depend on the vesicle size. ${ }^{10}$ Furthermore, $\mathrm{DC}_{8,9} \mathrm{PC}$ is classified as an identical chain $\mathrm{PC}$ that contains diacetylene in both acyl chains. Compared with mixed-chain PC that contains diacetylene group within only one hydrocarbon chain, the identical chain PC tends to exhibit lower conversion yields but easily produces the polymer network because a single molecule can potentially form links to four other neighbor lipid molecules. ${ }^{11}$ This local polymer network can trigger changes in a membrane's density and pore formation.

Phospholipid bilayer liposomes have always been of keen interest as microcapsules for drugs transports ${ }^{4}$ or functional particles ${ }^{12}$ and as models for biomembranes. ${ }^{1,2}$ Liposomes are found on a variety of length scales, with vesicle sizes ranging from small (nanometer) to giant (micrometer) andin a variety of structures, ranging from unilamellar to multilamellar structures. Although small unilamellar vesicles (SUVs) are often treated as bionanoparticles that are characterized by spectroscopy methods ${ }^{11,13}$ and are also used to fuse onto solid-substrate to form planar supported lipid bilayers, ${ }^{14}$ giant unilamellar vesicles (GUVs) are often observed by conventional microscopes, ${ }^{4,15}$ that is, fluorescence and confocal microscopes, and are used as membrane 
platforms to study protein-membrane interactions, ${ }^{16}$ especially interactions with some bulky proteins, for example, extracellular matrix proteins ${ }^{17}$ that can only be studied on GUVs system.

It has been known that the PCL reaction of polymerizable lipids within the liposomes are influenced by the size, ${ }^{10}$ lipid composition ${ }^{12}$ and phase (for example, gel or fluid) ${ }^{2,7,8}$ of liposomes. In the other words, the packing state of the hosting matrix strongly affects the kinetics and yield of the polymerization reaction in the membrane. However, vesicle systems cannot precisely control the packing state and the composition in each leaflet of the bilayers. ${ }^{18}$ The vesicle fusion method used to make the planar supported lipid bilayer is also restricted to the deposition of the fluid-phase lipids and cannot be used to create asymmetric bilayers. In this sense, the Langmuir monolayer is preferred as an ideal platform for fundamental studies because of its capability of precisely controlling the lateral packing and lipid composition. ${ }^{19}$ In this report, we systematically study the packing effect of the hosting matrices on the lipid-crosslinking reaction. The packing states are varied through the vesicle composition and the surface pressure of the Langmuir monolayers, thus obtaining the corresponding kinetics of the PCL reaction for each packing state.

\section{MATERIALS AND METHODS}

\section{Materials}

1,2-Dipalmitoyl-sn-glycero-3-phosphocholine (16:0 PC, DPPC), 1,2-dimyristoyl-sn-glycero-3-phosphocholine (14:0 PC, DMPC), 1,2-dioleoyl-sn-glycero-3phosphocholine (18:1 $\Delta 9$-Cis PC, DOPC) and 1,2-bis(10,12-tricosadiynoyl)sn-glycero-3-phosphocholine (23:2 Diyne $\mathrm{PC}, \mathrm{DC}_{8,9} \mathrm{PC}$ ) were purchased from Avanti Polar Lipids (Alabaster, AL, USA). 1,2-dihexadecanoyl-sn-glycero-3phosphoethanolamine, triethylammonium salt (Texas Red-DHPE) and Alexa ${ }^{448}$ Fluor dye were purchased from Molecular Probes (Eugene, OR, USA). Chloroform, sucrose and glucose were purchased from SigmaAldrich (St Louis, MO, USA).

\section{Preparation of liposomes}

SUV preparation. SUVs were prepared by hydration method. ${ }^{20}$ An amount of $1.5 \mathrm{mg}$ of lipid compositions of DPPC and $\mathrm{DC}_{8,9} \mathrm{PC}$ were placed in vial and chloroform was evaporated with nitrogen gas. Then, lipid multilayer films were dehydrated under vacuum for $1 \mathrm{~h}$. One millileter of $200 \mathrm{~mm}$ sucrose solution was added, and the solution was vortexed from $20 \mathrm{~min}$ above the transition temperature. Multilamellar vesicles were formed and were sonicated for $3 \mathrm{~min}$ to convert these vesicles into unilamellar vesicles.

GUV preparation. GUVs were formed by the electroformation method ${ }^{21}$ using a customized chamber that consisted of a pair of indium tin oxide glass sheets, $\mathrm{Cu}$ wires and a silicone spacer. The various compositions of DPPC, DMPC, DOPC and polymerizable lipid- $\mathrm{DC}_{8,9} \mathrm{PC}$ were dissolved in chloroform at a final concentration of $1.5 \mathrm{mg} \mathrm{ml}^{-1}$.22 The mixtures were doped with $0.5 \mathrm{~mol} \%$ Texas red-DHPE, allowing the lipid domain to be visualized by confocal microscopy. Lipid mixtures were spread onto the indium tin oxide glass to fabricate the thin film, and kept under vacuum for $30 \mathrm{~min}$. After evaporation of the solvent, the chambers were filled with $200 \mathrm{~mm}$ sucrose solution containing Alexa ${ }^{448}$ Fluor dye for the observation of porous structure. An alternating electric field ( $10 \mathrm{~Hz}, 1.5 \mathrm{Vpp}$, sine function) was applied to the indium tin oxide glass by an arbitrary function generator (Tektronix, Beaverton, OR, USA) for at least $3 \mathrm{~h}$. Subsequently, the frequency was decreased to $4 \mathrm{~Hz}$ and the amplitude was increased to $2.5 \mathrm{Vpp}$; a square function was then applied for $30 \mathrm{~min}$. GUV formation progressed above the transition temperature of lipids.

\section{Surface pressure measurement}

Surface pressure-area isotherms were recorded at a compression rate of $2 \mathrm{~cm}^{2} \mathrm{~min}^{-1}$ in a symmetric mode by using a Langmuir trough (611 M, NIMA Technology, The Science Park, Coventry, UK) containing a distilled water subphase. A mini Langmuir trough (302 M, NIMA) was used for the PCL reaction of $\mathrm{DC}_{8,9} \mathrm{PC}$ under UV exposure (VL-8.MC, Vilber Lourmat, $230 \mathrm{~V}$,
$50 / 60 \mathrm{~Hz}, 254 \mathrm{~nm}$, ZAC de Lamirault, Collégien) at a distance of $8 \mathrm{~cm}$. The trough was enclosed in a plexiglass box with black paper fully covered to prevent air vibration and to block the interfered lights. The entire trough setup was placed on an anti-vibration table to minimize the fluctuation caused by the operation of the instrument. A detailed description of the experiment setup and preparation is provided elsewhere. ${ }^{23}$ Briefly, after reaching the target pressures, the two barriers were adjusted automatically to maintain the pressures. This procedure was done until the area was constant, indicating that the monolayer was stable, and the barriers were stopped to fix the area. The UV lamp was immediately activated and the change of $\Pi$ was monitored for $\sim 200 \mathrm{~min}$. Upon the PCL reaction, the molecular area of $\mathrm{DC}_{8,9} \mathrm{PC}$ is supposed to be reduced, leading to the decrease in $\Pi$ because the area is fixed before the UV exposure.

\section{$\mathrm{UV}$ treatment for PCL reaction of $\mathrm{DC}_{8,9} \mathrm{PC}$ and $\mathrm{UV}$-vis spectral analysis}

For the PCL reaction, $1 \mathrm{ml}$ of the SUV solution was exposed to UV light at a distance of $6 \mathrm{~cm}$ for $1 \mathrm{~h}$ at room temperature. The color changes were captured by a digital camera. Visible and ultraviolet (UV-vis) spectroscopy was performed using a UV spectrophotometer (V-660, Jasco, Gauteng, South Africa) with quartz cells $(20 \mathrm{~mm} \times 20 \mathrm{~mm} \times 1 \mathrm{~mm})$. The polymerization reaction was monitored through its visible spectrum between 300 and $800 \mathrm{~nm}$. Upon irradiation of the $\mathrm{DC}_{8,9}$ PC-containing SUV aqueous dispersion with UV light, high-molecular-weight colored polymers were formed. The intensity of the color is associated with the amount of PCL reaction of $\mathrm{DC}_{8,9} \mathrm{PC}$ within the liposome.

\section{Confocal microscopy and fluorescence intensity measurement}

The leakage of the dye from GUVs was monitored by live confocal microscope (LSM410, Zeiss, Oberkochen, Germany). The 488-nm argon laser line and the 594-nm laser line were used for the excitation of Alexa ${ }^{448}$ Fluor and Texas red. The concentration of GUVs diluted 40-fold with the addition of a $200 \mathrm{~mm}$ glucose solution. Upon UV exposure, the fluorescence intensities of the GUV interior solution were monitored, and the background intensity of the glucose solution measured before the exposure was subtracted from the fluorescence intensity data. The fluorescence intensities were then normalized to the initial intensity value for comparison. Based on the decrease in the fluorescence intensity, the porous structure of the GUVs was confirmed, and the reaction rate was obtained as a function of time.

\section{RESULTS}

PCL reaction of $\mathrm{DC}_{8,9} \mathrm{PC}$ in SUVs

Upon the exposure of the $\mathrm{DC}_{8,9}$ PC-containing SUVs, which had a sufficient concentration of polymerizable lipids in the matrix of the SUVs, to UV light, the solution became reddish, indicating that polymers were formed. The intensity of color is associated with the degree of polymerization within the liposome and depends on several factors.

Two influence of two factors, the UV-exposed time and $\mathrm{DC}_{8,9} \mathrm{PC}$ fractions $(\Phi)$, is investigated for the occurrence and yield of polymerization reaction. As can be directly observed in Figure 1a, only DPPC-SUVs composed of $\Phi=10 \%$ exhibit reddish color and increased color intensity as a function of time. Figure $1 \mathrm{~b}$ shows a comparison of the UV absorption spectra for various $\Phi$ s, for example, $0,0.1,1$ and $10 \%$ in DPPC-SUVs. Maximum absorbance peaks appeared at $\sim 480$ and $525 \mathrm{~nm}$ only at $\Phi=10 \%$. The maximum absorbance wavelength depends on the number of the electronically coupled polymer units. Therefore, when the quantity of the coupled polymer units increases, a longer absorbance peak wavelength is obtained. Accordingly, the maximum absorbance peak that appeared at $480 \mathrm{~nm}$ corresponds to the shorter polymers, while the peak at $525 \mathrm{~nm}$ is assigned for a longer conjugation of cross bridges formed between the linear polymer through the glycerol of an identical chain PC (double, single and triple sequence of binding) ${ }^{24}$ The position of 
a

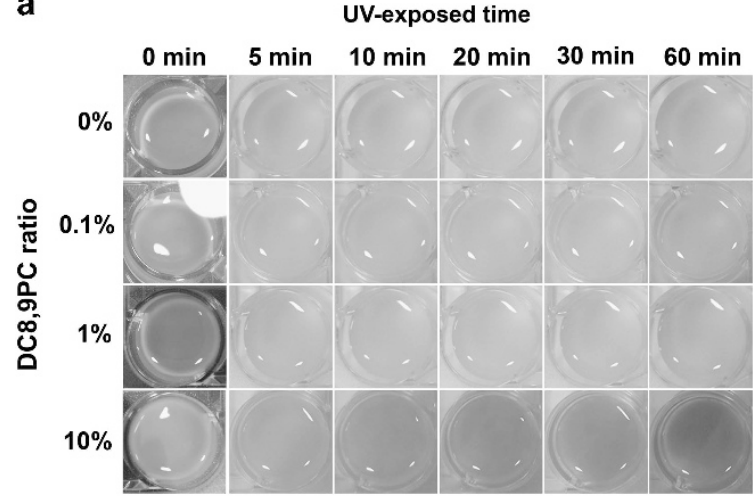

b

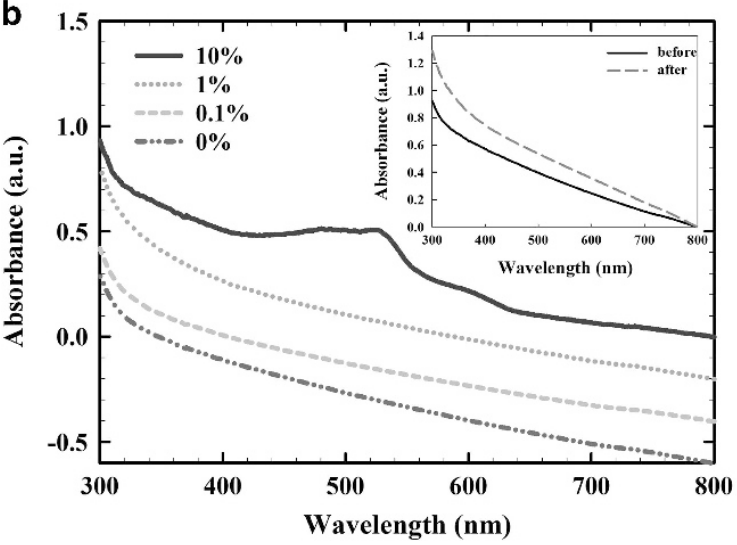

Figure 1 (a) Series of SUVs solution with different fractions of $D_{8,9} P C$ mixed with DPPC, under different UV exposure durations. (b) UV-vis absorption spectra of the corresponding SUVs solution in a. Inset: spectra of SUVs composed of an unsaturated lipid, DOPC, mixed with $10 \%$ of $\mathrm{DC}_{8,9} \mathrm{PC}$ before and after UV exposure. DPPC, 1,2-dipalmitoyl-sn-glycero-3phosphocholine; SUV, small unilamellar vesicles. A full color version of this figure is available at Polymer Journal online.

the peak is also in good agreement with reports in the literature. ${ }^{11}$ When the SUV matrices are composed of an unsaturated lipid, that is, DOPC, mixed with $10 \%$ of $\mathrm{DC}_{8,9} \mathrm{PC}$, no peak is observed, even though the spectrum taken after the UV exposure exhibits a higher overall intensity than that taken before the exposure, suggesting that no polymerization occurs in DOPC-SUVs. We note that both the electronically conjugated system on each $\mathrm{DC}_{8,9} \mathrm{PC}$ chain and the possibility of self-polymerizing between two the chains within the individual $\mathrm{DC}_{8,9} \mathrm{PC}$ molecule can enhance the UV absorption. However, the polymerization efficiency is not sufficient to form a peak for the case of a hosting matrix composed of unsaturated lipids.

\section{PCL reaction of $\mathrm{DC}_{8,9} \mathrm{PC}$ in GUVs}

The same experimental approach is applied for GUVs consisting of various lipids and $\Phi$ s. The microsize of a GUV and its capability to contain water-soluble fluorescent probes enable the morphological change of vesicles to be monitored by confocal microscopy; therefore, the pore formation induced by the PCL reaction of $\mathrm{DC}_{8,9} \mathrm{PC}$ can be elucidated by the presence of dye leakage from the inside to the outside. Figure 2 shows the direct visualization for vesicle morphology and the interior fluorescence intensity (green dye) before and after the UV exposure. Consistent with the results obtained for the SUVs, the GUVs composed of DOPC mixed with $10 \%$ of $\mathrm{DC}_{8,9} \mathrm{PC}$ also show no dye leakage (Figures 2a and $\mathrm{a}^{\prime}$ ) and also maintain the vesicle shapes after the UV exposure. We note that the brightness of the interior dye is always supposed to be slightly depleted after microscopic observation because of dye quenching. For the GUVs composed of DPPC (Figures $2 \mathrm{~b}$ and $\mathrm{b}^{\prime}$ ) and DMPC (Figures $2 \mathrm{c}$ and $\mathrm{c}^{\prime}$ ), the confocal images clearly show green dye leakage, and the leakage is more dramatic in the case of DPPC-GUV than in that of DMPC-GUV. During the duration of the experiment, DPPC-GUVs are found to shrink, with some even ruptured after $20 \mathrm{~min}$ of exposure to UV light. If the fluorescence quenching obtained from the DOPC case is used as the baseline, then the value of the fluorescence decay by UV treatment obtained in DPPC and DMPC vesicles are $\sim 90 \%$ and $78 \%$, respectively. These values are relatively correlated with the degree of polymerization.

Figure 3 shows the dye leaking efficiency of DPPC-GUVs mixed with various $\Phi$ s. $\Phi=0 \%$ (pure DPPC-GUVs) (Figures 3a and a') is used as the control sample that exhibits no dye leakage and the maintenance of vesicle shape. An almost identical leaking efficiency is observed for $\Phi=0.1 \%$, while for $\Phi \geqslant 1 \%$, the interior green dyes dramatically leak, and the vesicles are smaller and ruptured. To quantify the efficiency of dye leakage shown in Figure 3, the fluorescence intensity changes were normalized to the initial state and are presented in Figure 4. The pore formation is signified by an abrupt decrease in fluorescence intensity, shown in Figures $4 \mathrm{c}$ and $\mathrm{d}$ for $\Phi=1$ and $10 \%$, respectively. The intensity fluctuations in Figures $4 \mathrm{a}$ and $\mathrm{b}$, for pure DPPC and $\Phi=1 \%$ without UV exposure, respectively, are not due to dye leakage but are rather due to simple dye quenching and thermal diffusion caused by instrumental operation and UV light exposure (for Figure 4a). Because these factors affect the entire duration of the experiments, the normalization of the intensity by the initial state does not reduce the amplitude of intensity fluctuations. The values for the degree of polymerization can be estimated based on the dye intensity leakage, and are 8, 93 and $100 \%$ for $\Phi=0.1,1$, and $10 \%$, respectively.

In both SUV and GUV systems, DPPC functions well as a hosting matrix for the facilitation of the polymerization reaction within the liposome. Therefore, DPPC lipid was selected for further studies on the effect of packing states on the photo-polymerization of $\mathrm{DC}_{8,9} \mathrm{PCs}$ by mean of a Langmuir monolayer, as described in next section.

\section{PCL reaction of $\mathrm{DC}_{8,9} \mathrm{PC}$ in Langmuir monolayer}

According to the results for the vesicle systems, the PCL reaction of $\mathrm{DC}_{8,9} \mathrm{PC}$ depends on the amount of monomer and the saturation degree of the lipids comprising the liposome matrix. The unsaturated DOPC lipid does not induce polymerization, while the saturated lipids with different melting temperature $\left(T_{\mathrm{m}}\right)$, that is, DSPC (18:0 PC, $T_{\mathrm{m}} 55^{\circ} \mathrm{C}$, data not shown), DPPC (16:0 PC, $\left.T_{\mathrm{m}} 41^{\circ} \mathrm{C}\right)$ and DMPC (14:0 PC, $T_{\mathrm{m}} 24^{\circ} \mathrm{C}$ ), facilitate the PCL reaction of $\mathrm{DC}_{8,9} \mathrm{PC}$ resulting in the leakage of the interior dye. The basic differences between the unsaturated and saturated lipids lie in the packing ability and ordereddomain formation. Because the vesicle system fails to control the packing state, the Langmuir monolayer model, which precisely control the composition and packing state, ${ }^{19}$ has been employed to systematically study the effect of packing on the polymerization reaction within the monolayer film.

Figure 5 shows the $\Pi-A$ isotherms of mixed $D_{8,9}$ PC-DPPC monolayers at various $\Phi s$, ranging from 0 to $100 \%$. There are clear plateaus for pure DPPC and $\mathrm{DC}_{8,9} \mathrm{PC}$ profiles that are displayed as the elastic modulus peaks of the area compressibility diagram centered at 10 and $20 \mathrm{mN} \mathrm{m}^{-1}$, respectively (inset, Figure 5). The progressive depletion of plateaus observed for $\Phi$ in the 1-10\% range indicates that at these investigated $\Phi$, the two lipid components are well-mixed. 

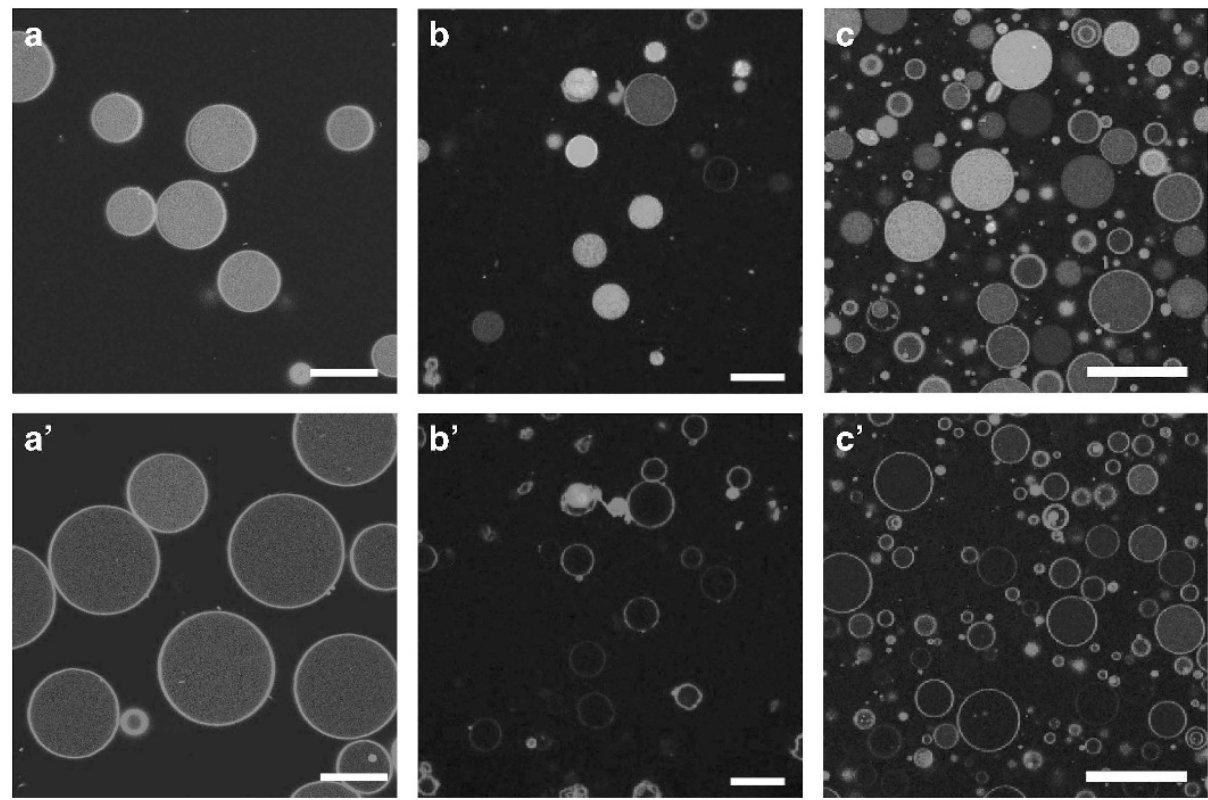

Scale bar $=\mathbf{5 0} \mu \mathrm{m}$

Figure 2 Direct visualization of dye leakage in GUVs because of the PCL reaction of $\mathrm{DC}_{8,9} \mathrm{PC}$ causing pore formation. Confocal microscopy of GUVs, filled with Alexa ${ }^{448}$ Fluor dye (green) and composed of $10 \% \mathrm{DC}_{8,9} \mathrm{PC}$ mixed with the unsaturated lipid, DOPC (a) or the saturated lipid, DPPC (b) or the saturated lipid with shorter hydrocarbon chain's length, DMPC (c), before (a-c) and after (a'-c') UV exposure. Scale bars, $50 \mu \mathrm{m}$. DPPC, 1,2-dipalmitoyl-sn-glycero-3phosphocholine; GUV, giant unilamellar vesicles; PCL, photo-crosslink. A full color version of this figure is available at Polymer Journal online.
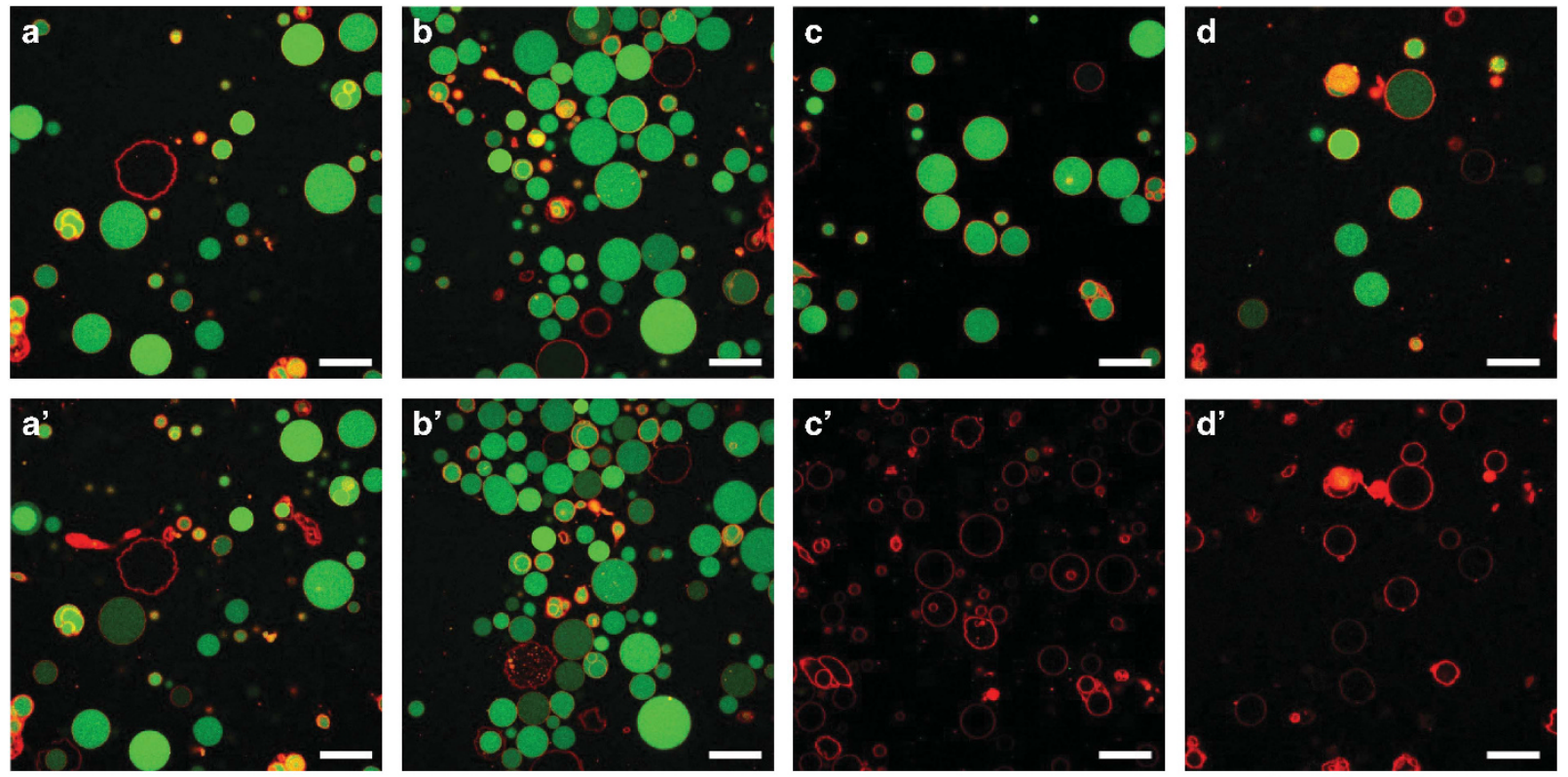

Scale bar $=\mathbf{5 0} \mu \mathrm{m}$

Figure 3 Direct visualization of dye leakage in GUVs due to PCL reaction of $\mathrm{DC}_{8,9} \mathrm{PC}$ causing pore formation. Confocal microscopy of GUVs which are filled with Alexa ${ }^{448}$ Fluor dye (green) and composed of $0 \%(\mathbf{a}), 0.1 \%(\mathbf{b}), 1 \%(\mathbf{c})$ and $10 \%$ (d) of DC 8,9 PC mixed with DPPC, before (a-d) and after (a'-d') UV exposure. DPPC, 1,2-dipalmitoyl-sn-glycero-3-phosphocholine; GUV, giant unilamellar vesicles; PCL, photo-crosslink.

Therefore, one can expect that $\mathrm{DC}_{8,9} \mathrm{PCs}$ are distributed in the liquidcondensed (LC) phases of DPPCs after the LC phases are formed.

Pore formation upon the PCL reaction of $\mathrm{DC}_{8,9} \mathrm{PC}$ has been well confirmed in GUVs by confocal microscopy. The pore can be understood as the ruptured spot in monolayer system, leading to a state with less packing. Therefore, the PCL reaction is expected to cause a decrease in $\Pi$, as observed in Figure 6 as a function of time.
Figure 6a shows the decrease of $\Pi$ with time for various $\Phi$ values, ranging from $0 \%$ (pure DPPC, control sample) to $10 \%$ at $30 \mathrm{mN} \mathrm{m}^{-1}$. Based on the $\Pi-A$ isotherms (Figure 5 ), we note that at this pressure for all compositions, the membrane monolayer is well-packed, and only the LC phase is supposed to exist. The decrease in $\Pi$ for the DPPC monolayer is simply accounted for by the thermal diffusion caused by the irradiation with the UV light. The $\Pi-t$ profile for 


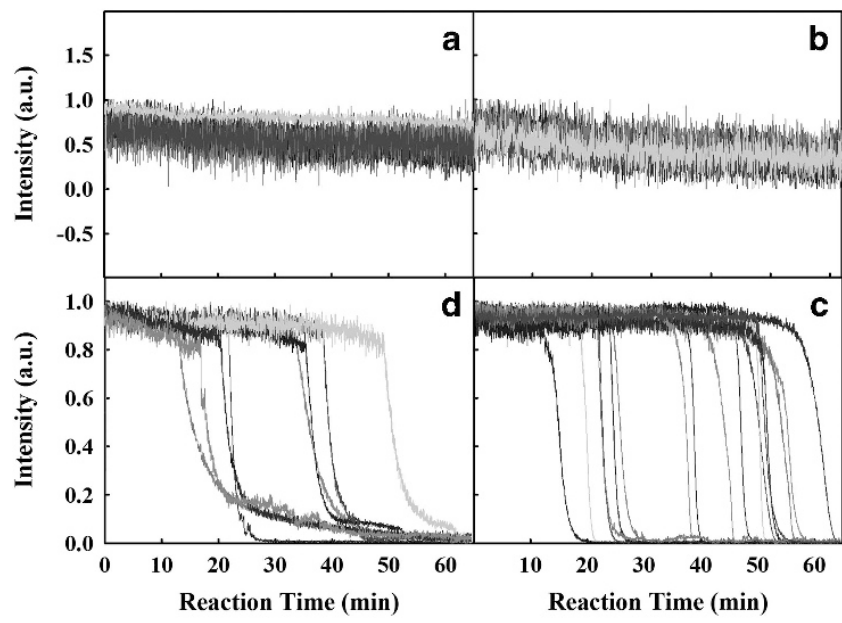

Figure 4 Time evolution of the fluorescence intensity changes of the GUVs for (a) pure DPPC $(\Phi=0 \%)$ with UV exposure, (b) $\Phi=1 \%$ without UV exposure, (c) $\Phi=1 \%$ with UV exposure and (d) $\Phi=10 \%$ with UV exposure. Diagrams of $\mathbf{a}$ and $\mathbf{b}$ are used as references. Diagrams of $\mathbf{a}, \mathbf{c}$ and $\mathbf{d}$ are deduced from the fluorescence intensity changes of the GUVs shown in Figures 3a, c and d, respectively. DPPC, 1,2-dipalmitoyl-sn-glycero-3phosphocholine; GUV, giant unilamellar vesicles. A full color version of this figure is available at Polymer Journal online.

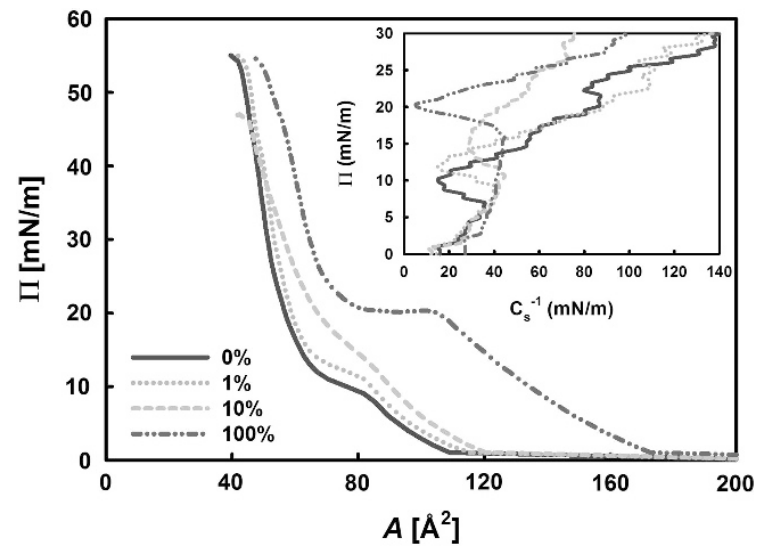

Figure $5 \Pi-A$ isotherms of mixed $\mathrm{DC}_{8,9} \mathrm{PC}$-DPPC monolayers at various $\Phi \mathrm{s}$, ranging from $\Phi=0$ to $100 \%$. Inset: elastic modulus of the area compressibility diagram of binary monolayers. DPPC, 1,2-dipalmitoyl-snglycero-3-phosphocholine. A full color version of this figure is available at Polymer Journal online.

$\Phi=1 \%$ is almost identical to that for the pure DPPC, suggesting that the polymerization reaction does not occur at $\Phi=1 \%$. However, for $\Phi \geqslant 5 \%$, the $\Pi$ values significantly decay as a function of time, indicating that the PCL reaction of $\mathrm{DC}_{8,9} \mathrm{PC}$ certainly occurs. The fact that the decrease in $\Pi$ is most obvious at $\Phi=10 \%$ suggests that the thermal diffusion term makes a smaller contribution than the kinetic term. Therefore, this system was selected to systematically study the effect of membrane packing on the crosslinking reaction. The results shown in Figure $6 \mathrm{~b}$ suggest that the reaction rate is strongly dependent on the lateral $\Pi$ of the film. Taken together with $\Pi-A$ isotherms (Figure 5), at $\Pi=5 \mathrm{mN} \mathrm{m}^{-1}$ the mixed DPPC monolayer is supposed to be in the liquid-expanded (LE) phase, and hardly any reaction is observed. Based on the comparison of the $\Pi-t$ profile of $\Pi=5 \mathrm{mN} \mathrm{m}^{-1}$ with that of pure DPPC and $\Phi=1 \%$, it can be concluded that the decay of $\Pi=5 \mathrm{mN} \mathrm{m}^{-1}$ is mostly attributed to the thermal diffusion of molecules. The DPPC monolayer at $\Pi=10 \mathrm{mN} \mathrm{m}^{-1}$ represents the transition from the LE phase to the LC phase and exhibits a plateau in the $\Pi-A$ isotherm (Figure 5). Because of the presence of LC domains, the decay rate at $\Pi=10 \mathrm{mN} \mathrm{m}^{-1}$ is significantly faster than that at $\Pi=5 \mathrm{mN} \mathrm{m}^{-1}$. Between $\Pi=10$ and $20 \mathrm{mN} \mathrm{m}^{-1}$, an increasing number of LC domains are formed, but the LE pool still exists, leading to a slow increase in the reaction rates. However, at $\Pi=30 \mathrm{mN} \mathrm{m}^{-1}$, a physiologically relevant pressure at which the lipid membrane is assumed to be fully packed in a condensed phase, the reaction rate is significantly increased. To quantitatively understand the packing effect, all $\Pi-t$ profiles are fitted to the kinetic law equations, and the kinetic parameters are described in detail below.

\section{Kinetics of $\mathrm{PCL}$ reaction of $\mathrm{DC}_{8,9} \mathrm{PC}$ in monolayer}

By monitoring the decrease of $\Pi$ as a function of the irradiation time, the apparent rate of polymerization is readily obtained. The $\Pi-t$ profiles are fit to

$$
\Delta \Pi(t)=\Pi(0)+a \exp (k t)
$$

where $\Delta \Pi(t)$ is the integrated surface pressure at time $t, a$ is the amplitude and $k$ is the rate of the PCL reaction of $\mathrm{DC}_{8,9} \mathrm{PC}$. A nonlinear least-squares analysis is employed to fit all profiles. The polymerization process is believed to occur by a single mechanism as indicated by an adequate fit to a first-order model (equation 1). The low $\Pi$ data are not well-fit, possibly because of the large contribution of thermal diffusion, while the fits show higher quality at high packing states because of the dominance of the polymerizing contribution. In addition to the kinetic rate constants, the half-life $\left(t_{1 / 2}\right)$ of the PCL reaction is also presented. The $t_{1 / 2}$, in terms of the rate of PCL reaction of $\mathrm{DC}_{8,9} \mathrm{PC}$ (equation 1), is given by

$$
t_{1 / 2}=\frac{\ln (2)}{2 k}
$$

Accordingly, the PCL reaction of $\mathrm{DC}_{8,9} \mathrm{PC}$ takes $\sim 385 \mathrm{~min}$ to reach $t_{1 / 2}$ in the LE state of monolayer $\left(\Pi=5 \mathrm{mN} \mathrm{m}^{-1}\right)$, while $t_{1 / 2}$ is reduced by more than fivefold, at $\sim 69 \mathrm{~min}$, in the homogeneous LC monolayer $\left(\Pi=30 \mathrm{mN} \mathrm{m}^{-1}\right)$. The kinetic parameters obtained from fitting is summarized in Table 1.

\section{DISCUSSION}

Polymerization reactions within self-assembled films, that is, lipid liposomes and monolayers, are strongly influenced by the hosting matrices. Understanding the influence of the matrices may lead to controlled reaction kinetics and yield, leading to significant impact on drug/gene delivery and controlled release systems. All data presented in this report are relevant for determination of the features of hosting matrices that govern the PCL reaction of the $\mathrm{DC}_{8,9} \mathrm{PC}$, photopolymerizable lipid, both qualitatively and quantitatively.

Based on our data, we hypothesize that within the range of the investigated $\mathrm{DC}_{8,9} \mathrm{PC}$ concentrations, the system is well-mixed with both saturated, that is, DMPC and DPPC, and unsaturated lipids, that is, DOPC in all membrane-mimicking systems. In other words, $\mathrm{DC}_{8,9} \mathrm{PCs}$ can either be embedded in the LC domains of DPPC or blended in the LE phase of DOPC. A PCL reaction of $\mathrm{DC}_{8,9} \mathrm{PCs}$ has not been observed when these are mixed with DOPC in liposomes, suggesting that the liquid phases (disordered phase) of the DOPC matrix keep $\mathrm{DC}_{8,9} \mathrm{PC}$ molecules far away from one another. On the other hand, the LC domains of DPPC in liposomes make the crossdistance between $\mathrm{DC}_{8,9} \mathrm{PC}$ molecules sufficiently close for the PCL reaction to proceed. It could be argued that the dramatic structural 


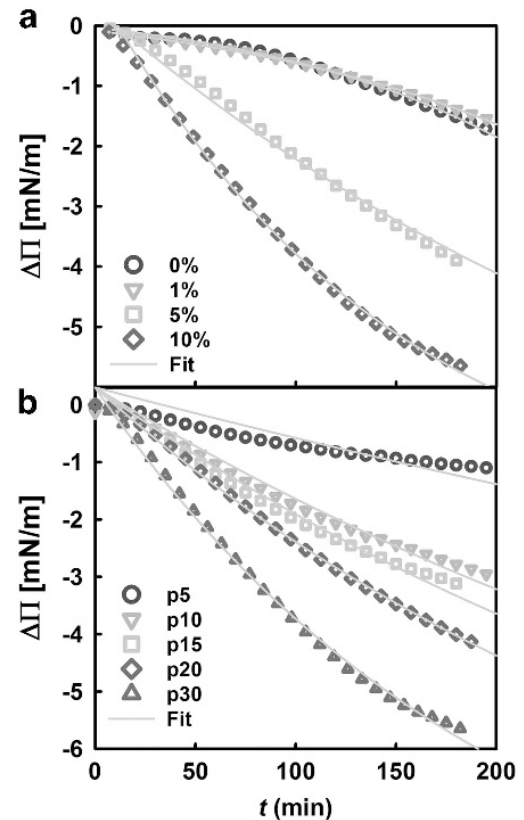

Figure $6 \mathrm{PCL}$ reaction of $\mathrm{DC}_{8,9} \mathrm{PC}$ in Langmuir monolayer. Changes of $\Pi$ are plotted versus time during UV exposure. (a) $\Delta \Pi-t$ for various $\mathrm{DC}_{8,9} \mathrm{PC}$ fractions mixed with DPPC and initial pressures were set at $30 \mathrm{mN} \mathrm{m}^{-1}$ before UV irradiation. (b) $\Delta \Pi-t$ for various initial $\Pi$ s for $\mathrm{DC}_{8,9} \mathrm{PC}$ fraction of $10 \%$. Solid lines are fits to the data using equation 1. DPPC, 1,2dipalmitoyl-sn-glycero-3-phosphocholine; PCL, photo-crosslink. A full color version of this figure is available at Polymer Journal online.

Table 1 Summary of PCL kinetics during UV exposure in DPPC monolayer as functions of $\mathrm{DC}_{8,9} \mathrm{PC}$ fraction $(\Phi)$ and surface pressure (II)

\begin{tabular}{lcccc}
\hline$\Phi(\%)$ & ${ }^{\mathrm{a}} \Pi_{i}\left(m \mathrm{~m}^{-1}\right)$ & ${ }^{\mathrm{b}} k\left(\mathrm{~min}^{-1}\right) \times 10^{-4}$ & $\ln (\mathrm{k})$ & $t_{1 / 2}(\mathrm{~min})$ \\
\hline 0 & 30 & $\mathrm{~N} / \mathrm{A}$ & $\mathrm{N} / \mathrm{A}$ & $\mathrm{N} / \mathrm{A}$ \\
1 & 30 & $\mathrm{~N} / \mathrm{A}$ & $\mathrm{N} / \mathrm{A}$ & $\mathrm{N} / \mathrm{A}$ \\
5 & 30 & $28.0 \pm 0.3$ & $-5.88 \pm 0.01$ & $123.8 \pm 1.2$ \\
10 & 5 & $9.0 \pm 0.4$ & $-7.01 \pm 0.04$ & $385.1 \pm 15.0$ \\
10 & 10 & $21.0 \pm 0.4$ & $-6.16 \pm 0.02$ & $165.0 \pm 3.9$ \\
10 & 15 & $24.0 \pm 0.3$ & $-6.03 \pm 0.01$ & $144.4 \pm 1.9$ \\
10 & 20 & $31.0 \pm 0.2$ & $-5.78 \pm 0.01$ & $111.8 \pm 0.7$ \\
10 & 30 & $50.0 \pm 0.3$ & $-5.30 \pm 0.01$ & $69.3 \pm 0.5$ \\
\hline
\end{tabular}

Abbreviations: DPPC, 1,2-dipalmitoyl-sn-glycero-3-phosphocholine; PCL, photo-crosslink

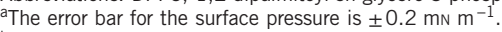

${ }^{\mathrm{b}}$ The error in the determined rate constant is s.e.m. provided from nonlinear regression fits

difference between DPPC and $\mathrm{DC}_{8,9} \mathrm{PC}$ may prevent their miscibility. However, Figure 5 shows that a small amount of $\mathrm{DC}_{8,9} \mathrm{PCs}$ can be well-mixed in the LC domains of DPPCs, even without being polymerized. Once $\mathrm{DC}_{8,9} \mathrm{PC}$ molecules are crosslinked, their packing states are more condensed, and hence, well-mixed state with LC domains of DPPC can be expected. This behavior of $\mathrm{DC}_{8,9} \mathrm{PCs}$ cannot be observed when they are mixed with DOPC because the dominant phase of the liposome is liquid, preventing the $\mathrm{DC}_{8,9} \mathrm{PCs}$ from getting close to one another. Although the possibility of a locally packed state of $\mathrm{DC}_{8,9} \mathrm{PCs}$ in the DOPC matrix cannot be excluded, it would be only as temporary packing state and cannot promote polymerization due to the $\mathrm{DC}_{8,9} \mathrm{PCs}$ ' cross-distance and molecular mobility.

The discrepancy in the critical $\Phi$ where the PCL reaction of $\mathrm{DC}_{8,9} \mathrm{PC}$ occurs in DPPC matrices between the SUVs (Figure 1) and

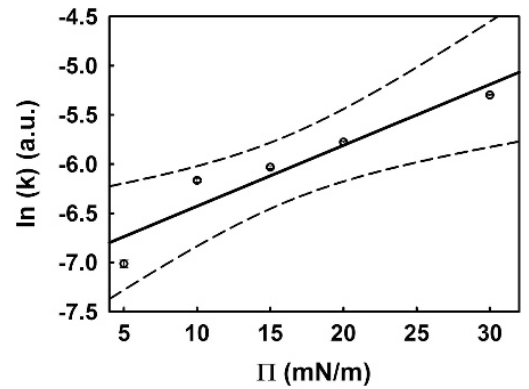

Figure 7 Plot of $\ln (k)$ versus surface pressure (П) for DPPC monolayer containing $\Phi=10 \%$. The linear least-squares fit to the data (solid line) and confidence bounds at 95\% (shaded dashed lines) are also shown. DPPC, 1,2-dipalmitoyl-sn-glycero-3-phosphocholine.

GUVs (Figure 3) is well expected because of the size difference and has been well observed in literature. ${ }^{10}$ However, one can raise a question as to exactly what occurs to facilitate the PCL reaction of $\mathrm{DC}_{8,9} \mathrm{PC}$ when the size of the liposomes composed of DPPC are increased. One possibility would be a capability of accommodating condenseddomains or inducing phase separation. We note that SUVs' size is in the nanometer range, which is supposed to be much smaller than a single condensed domain of the saturated lipid that would be formed, that is, DPPC. In this sense, the size of GUVs can easily accommodate the phase separation of the gel and liquid phases.

The most notable feature of the Langmuir monolayer is that the surface pressure is highly sensitive to the reaction, suggesting the PCL reaction of $\mathrm{DC}_{8,9} \mathrm{PC}$ has a strong impact on the packing state of the monolayer. With this high sensitivity, the present system is very promising for expanding this study to other types of photopolymerizable lipids for different applications. The kinetic data obtained in the monolayer system also enable the calculation of the pressure-dependent thermodynamics of activation based on the concepts of the transition state theory. ${ }^{25}$ The transition state theory theory was previously used by Anglin and Conboy to analyze the thermodynamics of phospholipid flip-flop ${ }^{26}$ on the PLSB systems. By similar approach, an activation area for the PCL process is determined by

$$
-R T\left(\frac{\partial \ln k}{\partial \Pi}\right)=\Delta a^{\ddagger}
$$

where $R$ is the gas constant, $t$ is the absolute temperature, and $\Delta a^{\ddagger}$ is activation area or the molecular area of the transition state. The measured rate $(k)$ of the lipid-crosslink as a function of initial $\Pi_{\mathrm{i}}$ from Table 1 can be used to calculate the $\Delta a^{\ddagger}$. Figure 7 shows a plot of $\ln (k)$ versus $\Pi$ and its fit to equation 3 using a linear least-square regression. From the slope of the resulting line, the activation area for the crosslinking reaction at $25^{\circ} \mathrm{C}$ is calculated to be $-25 \pm 5 \AA^{2}$ per molecule. We note that the additional heat from the UV lamp causes slight deviation, which is within the calculated uncertainty, and therefore can be ignored. The negative value of $\Delta a^{\ddagger}$ represents a decrease in the effective area of $25 \AA^{2}$ per molecule occupied by the transition state from an initial equilibrium area of two $\mathrm{DC}_{8,9} \mathrm{PC}$ molecules or two chains in an individual molecule. The local area that was decreased would consequently cause empty or ruptured spots on the membrane, leading to a decrease in $\Pi$, as observed in Figure 6. This phenomenon is expected, as each hydrocarbon chain of $\mathrm{DC}_{8,9} \mathrm{PC}$ has two diacetylene segments that would inhibit two chains or more than two molecules from reaching close proximity to each other. 
Therefore, the difference between the area occupied by the transition state and the initial area occupied by ground state is quite large.

To expand on this study, we wish to visualize the local rupture on the LC domains of the DPPC monolayer caused by the PCL process of $\mathrm{DC}_{8,9} \mathrm{PCs}$, and the population of ruptures is expected to increase as a function of $\mathrm{DC}_{8,9}$ PCs' concentration. This evidence would significantly strengthen our hypothesis that diacetylenecontaining lipids must be distributed in the LC phases of DPPC for polymerization to proceed.

\section{CONCLUSIONS}

We have demonstrated a systematic study on the effects of hosting matrices, for example, size, composition and lateral packing, on the PCL reaction of $\mathrm{DC}_{8,9} \mathrm{PC}$ within the membranes. The hosting matrices are required to have gel phase formation in the liposome or the LC phase in the monolayer in order for the PCL reaction of $\mathrm{DC}_{8,9} \mathrm{PCs}$ to proceed. The $\Pi-A$ isotherms show that $\mathrm{DC}_{8,9} \mathrm{PCs}$ are well-mixed with DPPCs within the investigated molar ratios, thus suggesting that $\mathrm{DC}_{8,9}$ PCs can be distributed in the LC phases of DPPC. We also found that the reaction rates were strongly dependent upon lateral pressures, or packing states, indicating the cross-distance between $\mathrm{DC}_{8,9} \mathrm{PCs}$ may have a main role in the PCL reaction. This observation can actually help explain why the reaction does not occur when $\mathrm{DC}_{8,9} \mathrm{PCs}$ are mixed with DOPC in liposomes because $\mathrm{DC}_{8,9} \mathrm{PCs}$ may be associated into the $\mathrm{LE}$ phase of $\mathrm{DOPC}$, which prevent $\mathrm{DC}_{8,9} \mathrm{PCs}$ from getting sufficiently close to one another so that the PCL reaction is not observed.

The monolayer study shows that the $\Pi-t$ decayed profiles are wellfitted by the nonlinear regression, and the fitting curves are matched to the first-order kinetic equation (equation 1), suggesting a unimolecular reaction. The activation area is also interpreted using the transition state theory. The obtained value for the area occupied by the transition state is likely the area reduced by the two molecules involved in polymerization, which causes a local rupture and leads to the decrease in lateral surface pressure.

\section{CONFLICT OF INTEREST}

The authors declare no conflict of interest.

\section{ACKNOWLEDGEMENTS}

This work was supported by the Mid-Career Researcher Program (grant no. 2011-0017539), the Nuclear Research R\&D Program and the Advanced Research Center for Nuclear Excellence and Basic Science Research Program (grant no. 2011-0031932) through the National Research Foundation of Korea funded by the Ministry of Science, Information and Communications Technology and Future Planning, Korea and Sogang University.
1 Ringsdorf, H., Schlarb, B. \& Venzmer, J. Molecular architecture and function of polymeric oriented systems: models for the study of organization, surface regconition, and dynamics of biomembranes. Angew Chem. Int. Ed. Engl. 27, 113-158 (1988).

2 Morigaki, K., Baumgart, T., Jonas, U., Offenhäusser, A. \& Knoll, W. Photopolymerization of diacetylene lipid bilayers and its application to the construction of micropatterned biomimetic membranes. Langmuir 18, 4082-4089 (2002).

3 Hub, H.-H., Hupfer, B., Koch, H. \& Ringsdorf, H. Polymerizable phospholipid analogues-new stable biomembrane and cell models. J. Angew Chem. Int. Ed. Engl. 19, 938-940 (1980).

4 Qin, G., Li, Z., Xia, R., Li, F., O'Neill, B. E., Goodwin, J. T., Khant, H. A., Chiu, W. \& Li, K. C. Partially polymerized liposomes: stable against leakage yet capable of instantaneous release for remote controlled drug delivery. Nanotechnology 22, 155605 (2011).

5 Schmidt, C., Mayer, M. \& Vogel, H. A chip-based biosensor for the functional analysis of single ion channels. Angew Chem. Int. Ed. Engl. 112, 3267-3270 (2000).

6 Wisniewski, N. \& Reichert, M. Methods for reducing biosensor membrane biofouling. Colloid Surface B 18, 197-219 (2000).

7 Mueller, A. \& O'Brien, D. F. Supramolecular materials via polymerization of mesophases of hydrated amphiphiles. Chem. Rev. 102, 727-758 (2002).

8 O'Brien, D. F., Armitage, B., Benedicto, A., Bennett, D. E., Lamparski, H. G., Lee, Y.-S., Srisiri, W. \& Sisson, T. M. Polymerization of preformed self-organized assemblies. Acc. Chem. Res. 31, 861-868 (1998).

9 Yager, P. \& Schoen, P. E. Formation of tubules by a polymerizable surfactant. Mol. Crys. Liq. Crys 106, 371-381 (1984).

10 Peek, B. M., Callahan, J. H., Namboodiri, K., Singh, A. \& Gaber, B. P. Effect of vesicle size on the polymerization of a diacetylene lipid. Macromolecules 27, 292-297 (1994).

11 Johnston, D. S., McLean, L. R., Whittam, M. A., Clark, A. D. \& Chapman, D. Spectra and physical properties of liposomes and monolayers of polymerizable phospholipids containing diacetylene groups in one or both acyl chains. Biochemistry 22, 3194-3202 (1983).

12 Yavlovich, A., Singh, A., Tarasov, S., Capala, J., Blumenthal, R. \& Puri, A. Design of liposomes containing photopolymerizable phospholipids for triggered release of contents. J. Therm. Anal. Calorim. 98, 97-104 (2009).

13 Lopez, E., O'Brien, D. F. \& Whitesides, T. H. Structural effects on the photopolymerization of bilayer membranes. J. Am. Chem. Soc. 104, 305-307 (1982).

14 Kim, H., Lee, K. Y., Ryu, S. R., Jung, K.-H., Ahn, T. K., Lee, Y., Kwon, O.-S., Park, S.-J., Parker, K. K. \& Shin, K. Charge-selective membrane protein patterning with proteoliposomes. R. Soc. Chem. Adv. 5, 5193-5191 (2015).

15 Kampf, J. P., Cupp, D. \& Kleinfeld, A. M. Different mechanisms of free fatty acid flip-flop and dissociation revealed by temperature and molecular species dependence of transport across lipid vesicles. J. Biol. Chem. 281, 21566-21574 (2006).

16 Tamba, Y. \& Yamazaki, M. Magainin 2-induced pore formation in the lipid membranes depends on its condentration in the membrane interface. J. Phys. Chem. B 113, 4846-4852 (2009).

17 Kahya, N. Protein-protein and protein-lipid interactions in domain-assembly: lessons from giant unilamellar vesicles. Biochim. Biophys. Acta 1798, 1392-1398 (2010).

18 Marsh, D. Lateral pressure in membranes. Biochim. Biophys. Acta 1286, 183-223 (1996).

19 Phan, M. D. \& Shin, K. A Langmuir monolayer: Ideal model membrane to study cell. J. Chem. Biol. Interfaces 2, 1-5 (2014).

20 Mueller, P., Chien, T. \& Rudy, B. Formation and properties of cell-size lipid bilayer vesicles. Biophys. J. 44, 375 (1983).

21 Angelova, M. I. \& Dimitrov, D. S. Liposome electroformation. Faraday Discuss. Chem. Soc. 81, 303-311 (1986).

22 Lopez, E., O'Brien, D. \& Whitesides, T. Effects of membrane composition and lipid structure on the photopolymerization of lipid diacetylenes in bilayer membranes. Biochim. Biophys. Acta 693, 437-443 (1982).

23 Phan, M. D. \& Shin, K. Effects of cardiolipin on membrane morphology: A Langmuir monolayer study. Biophys. J. 108, 1977-1986 (2015).

24 Alonso-Romanowski, S., Chiaramoni, N. S., Lioy, V. S., Gargini, R. A., Viera, L. I. \& Taira, M. C. Characterization of diacetylenic liposomes as carriers for oral vaccines. Chem. Phys. Lipid 122, 191-203 (2003).

25 Van Eldik, R., Asano, T. \& Le Noble, W. Activation and reaction volumes in solution.2. Chem. Rev. 89, 549-688 (1989).

26 Anglin, T. C. \& Conboy, J. C. Lateral pressure dependence of the phospholipid transmembrane diffusion rate in planar supported lipid bilayers. Biophys. J. 95 186-193 (2008). 